Undergraduate Texts in Mathematics

Editors

S. Axler

K.A. Ribet 
Abbott: Understanding Analysis.

Anglin: Mathematics: A Concise History and Philosophy.

Readings in Mathematics.

Anglin/Lambek: The Heritage of Thales.

Readings in Mathematics.

Apostol: Introduction to Analytic

Number Theory. Second edition.

Armstrong: Basic Topology.

Armstrong: Groups and Symmetry.

Axler: Linear Algebra Done Right. Second edition.

Beardon: Limits: A New Approach to Real Analysis.

Bak/Newman: Complex Analysis. Second edition.

Banchoff/Wermer: Linear Algebra Through Geometry. Second edition.

Berberian: A First Course in Real Analysis.

Bix: Conics and Cubics: A Concrete Introduction to Algebraic Curves.

Brémaud: An Introduction to Probabilistic Modeling.

Bressoud: Factorization and Primality Testing.

Bressoud: Second Year Calculus. Readings in Mathematics.

Brickman: Mathematical Introduction to Linear Programming and Game Theory.

Browder: Mathematical Analysis: An Introduction.

Buchmann: Introduction to Cryptography.

Buskes/van Rooij: Topological Spaces: From Distance to Neighborhood.

Callahan: The Geometry of Spacetime: An Introduction to Special and General Relavitity.

Carter/van Brunt: The LebesgueStieltjes Integral: A Practical Introduction.

Cederberg: A Course in Modern Geometries. Second edition.
Chambert-Loir: A Field Guide to Algebra

Childs: A Concrete Introduction to Higher Algebra. Second edition.

Chung/AitSahlia: Elementary Probability Theory: With Stochastic Processes and an Introduction to Mathematical Finance. Fourth edition.

Cox/Little/O'Shea: Ideals, Varieties, and Algorithms. Second edition.

Croom: Basic Concepts of Algebraic Topology.

Cull/Flahive/Robson: Difference Equations: From Rabbits to Chaos

Curtis: Linear Algebra: An Introductory Approach. Fourth edition.

Daepp/Gorkin: Reading, Writing, and Proving: A Closer Look at Mathematics.

Devlin: The Joy of Sets: Fundamentals of Contemporary Set Theory. Second edition.

Dixmier: General Topology.

Driver: Why Math?

Ebbinghaus/Flum/Thomas:

Mathematical Logic. Second edition.

Edgar: Measure, Topology, and Fractal Geometry.

Elaydi: An Introduction to Difference Equations. Third edition.

Erdõs/Surányi: Topics in the Theory of Numbers.

Estep: Practical Analysis in One Variable.

Exner: An Accompaniment to Higher Mathematics.

Exner: Inside Calculus.

Fine/Rosenberger: The Fundamental Theory of Algebra.

Fischer: Intermediate Real Analysis.

Flanigan/Kazdan: Calculus Two: Linear and Nonlinear Functions. Second edition.

Fleming: Functions of Several Variables. Second edition.

Foulds: Combinatorial Optimization for Undergraduates.

Foulds: Optimization Techniques: An Introduction. 
John Stillwell

\section{The Four Pillars of Geometry}

With 138 Illustrations

并 Springer 
John Stillwell

Department of Mathematics

University of San Francisco

San Francisco, CA 94117-1080

USA

stillwell@usfca.edu

\section{Editorial Board}

S. Axler

Mathematics Department

San Francisco State University

San Francisco, CA 94132

USA

\author{
K.A. Ribet \\ Department of Mathematics \\ University of California \\ at Berkeley \\ Berkeley, CA 94720-3840 \\ USA
}

Mathematics Subject Classification (2000): 51-xx, 15-xx

Library of Congress Control Number: 2005929630

ISBN-10: 0-387-25530-3

Printed on acid-free paper.

ISBN-13: 978-0387-25530-9

(C) 2005 Springer Science+Business Media, Inc.

All rights reserved. This work may not be translated or copied in whole or in part without the written permission of the publisher (Springer Science+Business Media, Inc., 233 Spring Street, New York, NY 10013, USA), except for brief excerpts in connection with reviews or scholarly analysis. Use in connection with any form of information storage and retrieval, electronic adaptation, computer software, or by similar or dissimilar methodology now known or hereafter developed is forbidden.

The use in this publication of trade names, trademarks, service marks, and similar terms, even if they are not identified as such, is not to be taken as an expression of opinion as to whether or not they are subject to proprietary rights.

Printed in the United States of America.

$\begin{array}{lllllllll}9 & 8 & 7 & 6 & 5 & 4 & 3 & 2 & 1\end{array}$

springeronline.com 
To Elaine 


\section{Preface}

Many people think there is only one "right" way to teach geometry. For two millennia, the "right" way was Euclid's way, and it is still good in many respects. But in the 1950s the cry "Down with triangles!" was heard in France and new geometry books appeared, packed with linear algebra but with no diagrams. Was this the new "right" way, or was the "right" way something else again, perhaps transformation groups?

In this book, I wish to show that geometry can be developed in four fundamentally different ways, and that all should be used if the subject is to be shown in all its splendor. Euclid-style construction and axiomatics seem the best way to start, but linear algebra smooths the later stages by replacing some tortuous arguments by simple calculations. And how can one avoid projective geometry? It not only explains why objects look the way they do; it also explains why geometry is entangled with algebra. Finally, one needs to know that there is not one geometry, but many, and transformation groups are the best way to distinguish between them.

Two chapters are devoted to each approach: The first is concrete and introductory, whereas the second is more abstract. Thus, the first chapter on Euclid is about straightedge and compass constructions; the second is about axioms and theorems. The first chapter on linear algebra is about coordinates; the second is about vector spaces and the inner product. The first chapter on projective geometry is about perspective drawing; the second is about axioms for projective planes. The first chapter on transformation groups gives examples of transformations; the second constructs the hyperbolic plane from the transformations of the real projective line.

I believe that students are shortchanged if they miss any of these four approaches to the subject. Geometry, of all subjects, should be about taking different viewpoints, and geometry is unique among the mathematical disciplines in its ability to look different from different angles. Some prefer 
to approach it visually, others algebraically, but the miracle is that they are all looking at the same thing. (It is as if one discovered that number theory need not use addition and multiplication, but could be based on, say, the exponential function.)

The many faces of geometry are not only a source of amazement and delight. They are also a great help to the learner and teacher. We all know that some students prefer to visualize, whereas others prefer to reason or to calculate. Geometry has something for everybody, and all students will find themselves building on their strengths at some times, and working to overcome weaknesses at other times. We also know that Euclid has some beautiful proofs, whereas other theorems are more beautifully proved by algebra. In the multifaceted approach, every theorem can be given an elegant proof, and theorems with radically different proofs can be viewed from different sides.

This book is based on the course Foundations of Geometry that I taught at the University of San Francisco in the spring of 2004. It should be possible to cover it all in a one-semester course, but if time is short, some sections or chapters can be omitted according to the taste of the instructor. For example, one could omit Chapter 6 or Chapter 8. (But with regret, I am sure!)

\section{Acknowledgements}

My thanks go to the students in the course, for feedback on my raw lecture notes, and especially to Gina Campagna and Aaron Keel, who contributed several improvements.

Thanks also go to my wife Elaine, who proofread the first version of the book, and to Robin Hartshorne, John Howe, Marc Ryser, Abe Shenitzer, and Michael Stillwell, who carefully read the revised version and saved me from many mathematical and stylistic errors.

Finally, I am grateful to the M. C. Escher Company - Baarn - Holland for permission to reproduce the Escher work Circle Limit I shown in Figure 8.19, and the explicit mathematical transformation of it shown in Figure 8.10. This work is copyright (2005) The M. C. Escher Company.

JOHN STILLWELL San Francisco, November 2004 South Melbourne, April 2005 


\section{Contents}

Preface vii

1 Straightedge and compass 1

1.1 Euclid's construction axioms . . . . . . . . . . . 2

1.2 Euclid's construction of the equilateral triangle . . . . . 4

1.3 Some basic constructions . . . . . . . . . . . . 6

1.4 Multiplication and division . . . . . . . . . . . . . . . 10

1.5 Similar triangles . . . . . . . . . . . . . . . . . 13

1.6 Discussion . . . . . . . . . . . . . . . . . 17

2 Euclid's approach to geometry 20

2.1 The parallel axiom . . . . . . . . . . . . . 21

2.2 Congruence axioms . . . . . . . . . . . . . . . . . . . . 24

2.3 Area and equality . . . . . . . . . . . . . 26

2.4 Area of parallelograms and triangles . . . . . . . . . . 29

2.5 The Pythagorean theorem . . . . . . . . . . . . . . 32

2.6 Proof of the Thales theorem . . . . . . . . . . . 34

2.7 Angles in a circle . . . . . . . . . . . . . . . 36

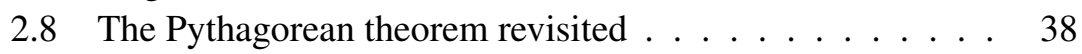

2.9 Discussion . . . . . . . . . . . . . . . . . . . 42

3 Coordinates 46

3.1 The number line and the number plane . . . . . . . . 47

3.2 Lines and their equations . . . . . . . . . . . . . 48

3.3 Distance . . . . . . . . . . . . . . . . 51

3.4 Intersections of lines and circles $\ldots \ldots \ldots 5$

3.5 Angle and slope . . . . . . . . . . . . . . . 55

3.6 Isometries . . . . . . . . . . . . . . . . . . . 57 
3.7 The three reflections theorem ........... 61

3.8 Discussion ................ 63

4 Vectors and Euclidean spaces $\quad 65$

4.1 Vectors ... . . . . . . . . . . . . 66

4.2 Direction and linear independence . . . . . . . . . . 69

4.3 Midpoints and centroids ............ . 71

4.4 The inner product ... . . . . . . . . . . . 74

4.5 Inner product and cosine . . . . . . . . . . . 77

4.6 The triangle inequality . . . . . . . . . . . 80

4.7 Rotations, matrices, and complex numbers . . . . . . . 83

4.8 Discussion ................. 86

5 Perspective $\quad 88$

5.1 Perspective drawing . . . . . . . . . . . . . . 89

5.2 Drawing with straightedge alone . . . . . . . . . . 92

5.3 Projective plane axioms and their models . . . . . . . . 94

5.4 Homogeneous coordinates . . . . . . . . . . . . 98

5.5 Projection ... . . . . . . . . . . . . 100

5.6 Linear fractional functions . . . . . . . . . . . . . 104

5.7 The cross-ratio . . . . . . . . . . . . . 108

5.8 What is special about the cross-ratio? . . . . . . . . 110

5.9 Discussion ................. 113

6 Projective planes 117

6.1 Pappus and Desargues revisited . . . . . . . . . . . 118

6.2 Coincidences ................ 121

6.3 Variations on the Desargues theorem . . . . . . . . 125

6.4 Projective arithmetic . . . . . . . . . . . . . 128

6.5 The field axioms . . . . . . . . . . . . 133

6.6 The associative laws .............. . . 136

6.7 The distributive law . . . . . . . . . . . . . 138

6.8 Discussion ................ 140

7 Transformations 143

7.1 The group of isometries of the plane . . . . . . . . . . . 144

7.2 Vector transformations . . . . . . . . . . . . 146

7.3 Transformations of the projective line . . . . . . . 151

7.4 Spherical geometry .............. 154 
7.5 The rotation group of the sphere . . . . . . . . . . . 157

7.6 Representing space rotations by quaternions . . . . . . . 159

7.7 A finite group of space rotations . . . . . . . . . . 163

7.8 The groups $\mathbb{S}^{3}$ and $\mathbb{R}^{3} \ldots \ldots \ldots$. . . . . . . . 167

7.9 Discussion . . . . . . . . . . . . . . . . . 170

8 Non-Euclidean geometry 174

8.1 Extending the projective line to a plane . . . . . . . . 175

8.2 Complex conjugation . . . . . . . . . . . . . 178

8.3 Reflections and Möbius transformations . . . . . . . 182

8.4 Preserving non-Euclidean lines . . . . . . . . . . . . . . 184

8.5 Preserving angle . . . . . . . . . . . . . 186

8.6 Non-Euclidean distance . . . . . . . . . . . . . . . . . . 191

8.7 Non-Euclidean translations and rotations . . . . . . . . 196

8.8 Three reflections or two involutions . . . . . . . . . . 199

8.9 Discussion . . . . . . . . . . . . . . . . . . 203

$\begin{array}{ll}\text { References } & 213\end{array}$

$\begin{array}{ll}\text { Index } & 215\end{array}$ 